\section{DECEMBER 2003}

- Forest Pest Management Forum 2003, 2-4 December.

Ottawa, Ontario. Contact: http://forum.glfc. forestry.ca

- 4th International Teak Conference (IUFRO), 2-5 December.

Peechi India (originally scheduled for 2002). Contact: K.M. Bhat: Fax: 91-487282249; kmbhat@kfri.org; http:/kfri.org/ html/k0500frm.html

- Woodworking Korea, 12-15 December.

Seoul, Republic of Korea. Contact: Reed Exhibitions (Germany): Fax: 49-211556231; rec.germany@reedexpo.co.uk; www.reedexpo.com

\section{FEBRUARY 2004}

- Annual Conference, International Society of Tropical Foresters, 4 February.

Washington, D.C. Contact: Dr. Les Whitmore, President, ISTF, 5400 Grosvenor Lane, Bethesda, MD 20814 USA. Fax: 301-897-3690; istf.Bethesda@verizon.net; www.istf-bethesda.org

\section{MARCH 2004}

- The Species at Risk: Pathways to Recovery Conference, 2-6 March. Victoria, BC, Canada. Contact: Patrick Daigle, RPF: Fax: 250-356-9145; patrick.daigle@gems3.gov.bc.ca; www. speciesatrisk2004.ca

- World of Wood, 17-19 March. Orlando, Florida USA. Contact: Fax: 703-820-8550; infor@ipawood.org; www.iwpawood.org/convention.html

- Wood Technology Clinic and Show, 17-19 March.

Portland, Oregon. Contact: Kathy Porter: Fax: 770-956-7938; kporter@mfi.com

- Human Dimensions of Family and Farm Forestry International Symposium, 29 March-1 April.

Washington State University, Contact: familyforestry@wsu.edu; www.familyforestrysymposium.wsu.edu

- AusTimber 2004, 29 March-3 April. Albury, NSW Australia. Contact: www.austimber2004.com

\section{APRIL 2004}

$\checkmark$ Management of Tropical Dry Forest Woodlands and Savanas-Assessment, Silviculture, and Scenarios (IUFRO), 12-14 April.

Brasilia, Brazil. Contact: Prof. Jose Imana Encinas: Fax: 55-61-347-0631; imana@ guarany.edp.unb.br

- 3rd International Symposium on Sustainable Management of Forest Resources-SIMFOR 2004, 21-23 April. Pinar del Rio, Cuba. Contact: C. Fernando Hernandez Martinez; Fax: 82-779353; fhernandez@ af.upr.edu.cu

- FOREXPO 2004, The European Forestry and Forest Management Trade Fair, 21-24 April.

Mimizan, France. Contact: Fax: 33-5-5681-78-98; info@forexpo.fr; www.forexpo.fr

\section{MAY 2004}

$\checkmark$ Northbound to Superior-Protecting Ontario's Nature: 73rd Annual General Meeting and Gathering, 9 May.

Toronto, Ontario. Contact: Tel: 1-800-4402366; Fax: 416-444-9866; info@ontarionature.org; www.ontarionature.org

\section{JUNE 2004}

- 8th International Timber Tradeshow for Professionals in the Wood Industry, 2-4 June.

Nantes, France. Contact: Samantha Padden: Fax: 011.33240730301 , sam@timbershow.com; www.timbershow.com

- Forest Genetics and Climate Change, 26-29 June.

Vernon, B.C. Canada. Contact: Alvin Yanchuk; Fax: 250-387-0046; Alvin.yanchuk@gems4.gov.bc.ca

- 1st World Congress of Agroforestry: Working Together for Sustainable Land-use Systems. 27 June-2 July. Orlando, Florida, USA. Contact: Mandy Padgett: mrpadgett@mail.ifas.ufl.edu; http://conference.ifas.ufl.edu/wca

\section{JULY 2004}

- Working Together for Sustainable Land-use Systems: 1st World Congress of Agroforestry 2004, 27 June-4 July. Orlando, Florida. Contact: http://conference.ifas.ufl.edu/wca
- 36th Session of the International Tropical Timber Council, 20-23 July. Switzerland. Contact: Collins Ahadome: Fax: 81-45-223-1111; itto@itto.orjp; www.itto.or.jp

- 7th INTECOL International Wetlands Conference, 25-31 July.

Utrecht, The Netherlands. Contact: congresbureau2@fbu.uu.nl; www.bio.uu.nl/ intecol

\section{AUGUST 2004}

- XXII IUFRO World CongressForests in the Balance: Linking Tradition and Technology, 8-13 August

- Forest Diversity and Resistance to Native and Exotic Pest Insects (IUFRO), 15-20 August. Hammer Springs, New Zealand. Contact: Andrew Liebhold; Fax: 304-285-1505; aliebhold@fs.fed.us; http://iufro.boku.ac.at/iufro

- International Congress of Entomology, 15-21 August.

Brisbane, Australia. Contact: Ashley Gordon, Congress Director: Ashley@ ccm.com.au; www.ccm.com.au/icoe/

\section{SEPTEMBER 2004}

- The Use of Vegetation to Improve Slope Stability (IUFRO), 6-10 September. Thessaloniki, Greece. Contact:Sanna Dupuy: Fax:33-5-566-80713; ecoconf@ irbb3. pierroton.inra.fr

$\checkmark$ Regenerating Mountain Forests, 12-16 September.

Kloster Seeon, Bavaria. Contact: Dr. Peter Brang: Fax: 41 (0) 173922 15; www.forst. tumuenchen.de/ext/lst/waldb/iufro/index en.html

- DEMO International 2004, 16-18 September.

Quebec City, Quebec, Canada. Contact: Anne Sawyer, Canadian Woodlands Forum; Tel: 514-392-6947; asawyer@cwfcof.org

\section{OCTOBER 2004}

$\checkmark$ New Tools for the Urban Forestry Toolbox: 6th Canadian Urban Forest Conference (CUFC), October (dates TBA). Vancouver, B.C. Contacts: Christian Walli, 6th Canadian Urban Forest Conference, tel.604-521-7771; christian_walli@brinkman. ca; Michael Rosen, Tree Canada Foundation, tel. 613-567-5545; mrosen@ treecanada.ca 